\title{
Co-cultivation and transcriptome sequencing of two co-existing fish pathogens Moritella viscosa and Aliivibrio wodanis
}

\author{
Erik Hjerde ${ }^{1}$, Christian Karlsen ${ }^{2}$, Henning Sørum ${ }^{2}$, Julian Parkhill ${ }^{3}$, Nils Peder Willassen ${ }^{1,4^{*}}$ and Nicholas R. Thomson ${ }^{3^{*}}$
}

\begin{abstract}
Background: Aliivibrio wodanis and Moritella viscosa have often been isolated concurrently from fish with winterulcer disease. Little is known about the interaction between the two bacterial species and how the presence of one bacterial species affects the behaviour of the other.

Results: The impact on bacterial growth in co-culture was investigated in vitro, and the presence of $A$. wodanis has an inhibitorial effect on M. viscosa. Further, we have sequenced the complete genomes of these two marine Gram-negative species, and have performed transcriptome analysis of the bacterial gene expression levels from in vivo samples. Using bacterial implants in the fish abdomen, we demonstrate that the presence of $A$. wodanis is altering the gene expression levels of $M$. viscosa compared to when the bacteria are implanted separately.

Conclusions: From expression profiling of the transcriptomes, it is evident that the presence of $A$. wodanis is altering the global gene expression of $M$. viscosa. Co-cultivation studies showed that $A$. wodanis is impeding the growth of $M$. viscosa, and that the inhibitorial effect is not contact-dependent.
\end{abstract}

Keywords: Aliivibrio, Moritella, Complete genome, RNA sequencing, Co-culture, Co-infection, Bacteriocin, Winter-ulcer

\section{Background}

Winter-ulcer disease affects reared salmonids when seawater temperatures drop below $8{ }^{\circ} \mathrm{C}$ [1]. Moritella viscosa is the aetiological agent of the disease. The significance of Aliivibrio wodanis, which is often co-isolated with $M$. viscosa or as the only isolate from diseased fish is uncertain [2]. Both M. viscosa and A. wodanis are Gramnegative gammaproteobacteria. The Moritella genus is a member of the Moritellaceae family, and consists mainly of psychrophilic and barophilic species isolated from deep-sea or marine sediments. M. viscosa is the only currently known pathogenic member of the Moritella genus. $A$. wodanis belongs to the Aliivibrio genus of the Vibrionaceae family. Several species from this family are pathogens causing diseases in different marine animals. A. wodanis

\footnotetext{
* Correspondence: nils-peder.willassen@uit.no; nrt@sanger.ac.uk

${ }^{1}$ Department of Chemistry, Faculty of Science and Technology, University of

Tromsø, N-9037 Tromsø, Norway

${ }^{3}$ The Pathogen Sequencing Unit, The Wellcome Trust Sanger Institute,

Wellcome Trust Genome Campus, Hinxton, Cambridge CB10 1SA, UK

Full list of author information is available at the end of the article
}

will be the second Aliivibrio species with a complete genome available.

Both $M$. viscosa and $A$. wodanis are cytotoxic to fish cells [3]. M. viscosa 06/09/139 and A. wodanis 06/09/139, which are genome sequenced in this study, are also both separately producing clinically disease symptoms in Atlantic salmon Salmo salar in bath challenge and are able to coinfect Atlantic salmon in a bath co-infection model [3]. The role of this bacterial interplay in the pathogenesis of winterulcer is not known, but $A$. wodanis colonisation of fish surfaces is hypothesized to influence the progression of a $M$. viscosa infection.

Since the two bacteria often co-exist in ulcers and internal organs, it is likely that they interact with each other. Interspecies interactions have been extensively explored within oral microbial communities (reviewed in [4] and [5]). Similarly, interactions in multimicrobial communities in fish hosts might be beneficial through 
synergistic effects (e.g., utilization of metabolic products, biofilm formation) communication (e.g., quorum sensing), or competition (e.g., for nutritional resources).

A coordinated behaviour involving intercellular communication between two species could be regulated via the production and response to signal molecules [6]. This cell-density dependent regulation termed quorum sensing (QS), is likely to provide a selective advantage for both bacterial populations by allowing them to alter their morphology and physiology quickly to adapt to environmental changes. Many Gram-negative bacteria use $\mathrm{N}$-acyl homoserine lactones (AHLs) as QS signal molecules, and AHLs from one species can regulate the behaviour of another species. For example Escherichia coli are able detect and regulate gene transcription in response to AHLs without having the ability to produce the molecule itself [7]. In addition to AHLs, many Gram-negative bacteria produce and recognize an autoinducer called AI-2. The widespread existence of this signal molecule indicate that bacteria can communicate across species boundaries using AI-2 [8].

On the other hand, within a mixed bacterial community the bacteria may compete with their neighbours for space and resources. There are several mechanisms by which bacterial species can dominate and outcompete other organisms, but nutritional resources seems to be a focal point of microbial competition (reviewed in [9]). The ability to grow faster or to acquire nutrition more efficiently by one organism comes at the expense of another. For example, an important mechanism for the uptake of iron is mediated through siderophores. Siderophores are iron-scavenging molecules that are produced and secreted from the bacterial cells, and actively transported back into the cells when they have sequestered iron from the surrounding environment. The structure of over hundred terrestrial siderophores with different affinity to bind iron has been described. On the other hand, the structure of only a few marine siderophores is known, the majority belong to the family of amphiphiles. Many amphiphilic siderophores are anchored to the cell through one or more fatty acid appendages, which is an adaptation to the low abundance of iron in seawater [10]. It may also be advantageous for bacteria living in different environments, e.g., the seawater and inside a host, to be able to utilize different siderophores. Further, one bacterial species could be outcompeted by the presence of another that produces a high-affinity siderophore [11].

Bacteria can also inhibit growth and kill competitors by secreting antimicrobial compounds toxic to other bacteria. Bacteriocins are proteinaceous molecules that inhibit growth of similar or closely related bacteria species through lethal disruption of membrane potential and integrity [12]. Some bacteriocins like vibriocins produced by several members of the Vibrionaceae family have toxic effects on more distantly related bacteria [13]. In Gram-negative bacteria a conserved leader sequence termed the GG-motif [M(R/K)ELX3E(I/L)X2(I/V)XG(G/A)] has been determined in the $\mathrm{N}$-terminal part of bacteriocins [14]. This leader sequence is cleaved off by a transport peptidase which is often found together in the bacteriocin locus along with a HlyD family protein involved in the transport across the membrane [15]. Self-protection against the bacteriocin are thought to be encoded by abortive infection $(a b i)$ genes which also can be found in the bacteriocin locus [16].

To investigate if there exist any synergistic effects between $M$. viscosa and $A$. wodanis during the course of an infection, we first performed in vitro mono- and coculture growth studies in different media. We then applied Illumina technology to perform high-throughput sequencing of single-stranded cDNA from the transcriptomes of $M$. viscosa and $A$. wodanis. The RNA samples were collected from in vivo challenge studies on fish where the bacteria were grown in separate semi-permeable tubings in the abdomen, either individually or together in the same fish. Our results show that the gene expression levels and the growth of both bacteria alters from single culture and single infection to co-culture and co-infections, and that the changes are most profound for M. viscosa.

\section{Results}

\section{General features of the genomes}

The general features of the $M$. viscosa 06/09/139 and $A$. wodanis 06/09/139 genomes are summarized in Table 1 and graphically presented in Additional file 1 . The genome of $M$. viscosa has one chromosome $(5.1 \mathrm{Mb})$ and two small cryptic plasmids named pMVIS41 $(4.1 \mathrm{~kb})$ and pMVIS39 (3.9 kb). The overall GC content is $39.4 \%$. The genome of $A$. wodanis has two chromosomes $(3.0 \mathrm{Mb}$ and $1.5 \mathrm{Mb})$, and four plasmids named pAWOD920 (92 kb), pAWOD150 (15 kb), pAWOD72 (7.2 kb) and pAWOD19 (2.1 kb). The overall GC content is $38.5 \%$.

\section{Growth and co-cultivation}

The in vitro growth of the two bacteria was determined in both mono- and co-cultures. Mono-cultures of $A$. wodanis are growing faster than $M$. viscosa under experimental tube-settings used in this study (Fig. 1). Typical growth conditions utilizing broth in shaked culture flasks (Additional file 2) confirmed that A. wodanis grows faster than $M$. viscosa at $7{ }^{\circ} \mathrm{C}$. An increase of $\mathrm{NaCl}$ in the growth medium from $1.0 \%$ to $3.5 \%$ produced a distinct change in the growth kinetic of $M$. viscosa. A higher growth rate was observed for M. viscosa at $3.5 \%$ $\mathrm{NaCl}$. M. viscosa growth is inhibited when grown in cocultivation with $A$. wodanis. $M$. viscosa kept separate from $A$. wodanis by semipermeable tubings in the same 
Table 1 General genome features of M. viscosa 06/09/139 and A. wodanis 06/09/139

\begin{tabular}{|c|c|c|c|c|c|c|c|c|c|c|c|c|}
\hline & $\begin{array}{l}\text { Genome } \\
\text { size (bp) }\end{array}$ & $G+C(\%)$ & $\begin{array}{l}\text { \# of } \\
\text { CDSs }\end{array}$ & Coding (\%) & $\begin{array}{l}\text { \# of rRNA } \\
\text { operons }\end{array}$ & $\begin{array}{l}\text { \# of } \\
\text { tRNAs }\end{array}$ & $\begin{array}{l}\text { \# of } \\
\text { SRNAs }\end{array}$ & $\begin{array}{l}\text { CDSs with } \\
\text { TMHs }\end{array}$ & $\begin{array}{l}\text { CDSs with } \\
\text { signal peptides }\end{array}$ & $\begin{array}{l}\text { Putative } \\
\text { uncharacterized } \\
\text { proteins }\end{array}$ & $\begin{array}{l}\text { Pseudo/ } \\
\text { partial } \\
\text { CDSs }\end{array}$ & $\begin{array}{l}\text { IS } \\
\text { elements }\end{array}$ \\
\hline $\begin{array}{l}\text { M. viscosa 06/ } \\
09 / 139\end{array}$ & 5093989 & 39.43 & 4445 & 83.7 & 11 & 131 & 261 & 1076 & 728 & 634 & 163 & 49 \\
\hline Chromosome & 5086074 & 39.44 & 4436 & 83.8 & 11 & 131 & 261 & 1074 & 727 & 630 & 163 & 49 \\
\hline $\begin{array}{l}\text { Plasmid } \\
\text { (pMVIS41) }\end{array}$ & 4059 & 31.21 & 5 & 63.0 & 0 & 0 & 0 & 0 & 0 & 1 & 0 & 0 \\
\hline $\begin{array}{l}\text { Plasmid } \\
\text { (pMVIS39) }\end{array}$ & 3856 & 36.36 & 4 & 35.9 & 0 & 0 & 0 & 2 & 1 & 3 & 0 & 0 \\
\hline $\begin{array}{l}\text { A. wodanis 06/ } \\
09 / 139\end{array}$ & 4635126 & 38.48 & 4079 & 86.9 & 7 & 88 & 95 & 1048 & 1185 & 546 & 104 & 27 \\
\hline Chromosome I & 3003353 & 38.88 & 2640 & 87.3 & 6 & 69 & 69 & 658 & 519 & 280 & 48 & 16 \\
\hline Chromosome II & 1515310 & 37.72 & 1313 & 87.0 & 1 & 19 & 19 & 369 & 650 & 222 & 46 & 4 \\
\hline $\begin{array}{l}\text { Plasmid } \\
\text { (pAWOD920) }\end{array}$ & 91951 & 38.33 & 96 & 80.4 & 0 & 0 & 7 & 20 & 16 & 26 & 7 & 7 \\
\hline $\begin{array}{l}\text { Plasmid } \\
\text { (pAWOD150) }\end{array}$ & 15266 & 36.22 & 18 & 72.0 & 0 & 0 & 0 & 1 & 0 & 12 & 1 & 0 \\
\hline $\begin{array}{l}\text { Plasmid } \\
\text { (pAWOD72) }\end{array}$ & 7177 & 34.86 & 9 & 69.7 & 0 & 0 & 0 & 0 & 0 & 5 & 0 & 0 \\
\hline $\begin{array}{l}\text { Plasmid } \\
\text { (pAWOD19) }\end{array}$ & 2069 & 37.46 & 3 & 78.7 & 0 & 0 & 0 & 0 & 0 & 1 & 0 & 0 \\
\hline
\end{tabular}

cultivation system produced a lower growth rate compared to when $M$. viscosa where cultivated in semipermeable tubings alone (Fig. 1). Co-cultivation exerted less influence on $A$. wodanis. Growth development in a colony co-culture showed that the growth of $M$. viscosa is clearly inhibited in co-culture with $A$. wodanis (Fig. 2). The inhibitorial effect is observed in all relative initial inocula-ratios tested between $M$. viscosa and $A$. wodanis from 50:50 down to 99:1. Growth inhibition or survival of $M$. viscosa corresponds to the initial M. viscosa : A. wodanis proportion i.e., $M$. viscosa is more strongly affected in high $A$. wodanis-proportion co-culture colonies. In the same co-culture colonies the growth of $A$. wodanis increases until day 5 before a decrease in cfu's are observed, similar to the $A$. wodanis colony reference.

\section{Transcriptomics}

At the start of the experiment three biological replicates (three salmons) were used for each sample condition. Unfortunately the fish mortality rate was high and we

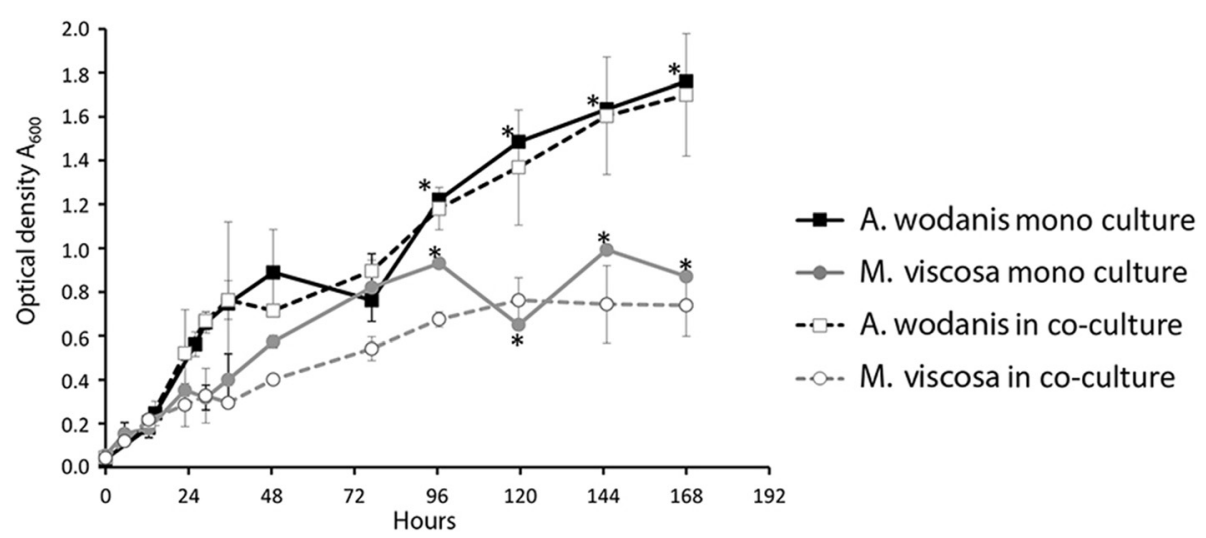

Fig. 1 Aliivibrio wodanis and Moritella viscosa in vitro growth curves. Mono-cultures of A. wodanis grows faster than M. viscosa, and A. wodanis inhibits $M$. viscosa growth when cultured in co-culture. The bacteria were grown in tubings; either as a mono-culture or in two separate tubings within the same culture system (co-cultivated). The growth was measured in optical density ( $\left.\mathrm{A}_{600}\right)$ and is presented as the average \pm standard deviation of three parallell experiments. The symbol * present at mono-culture sample time points from 96 to $168 \mathrm{~h}$ indicate $\mathrm{A}_{600}$ values obtained from only a single culture experiment representative 


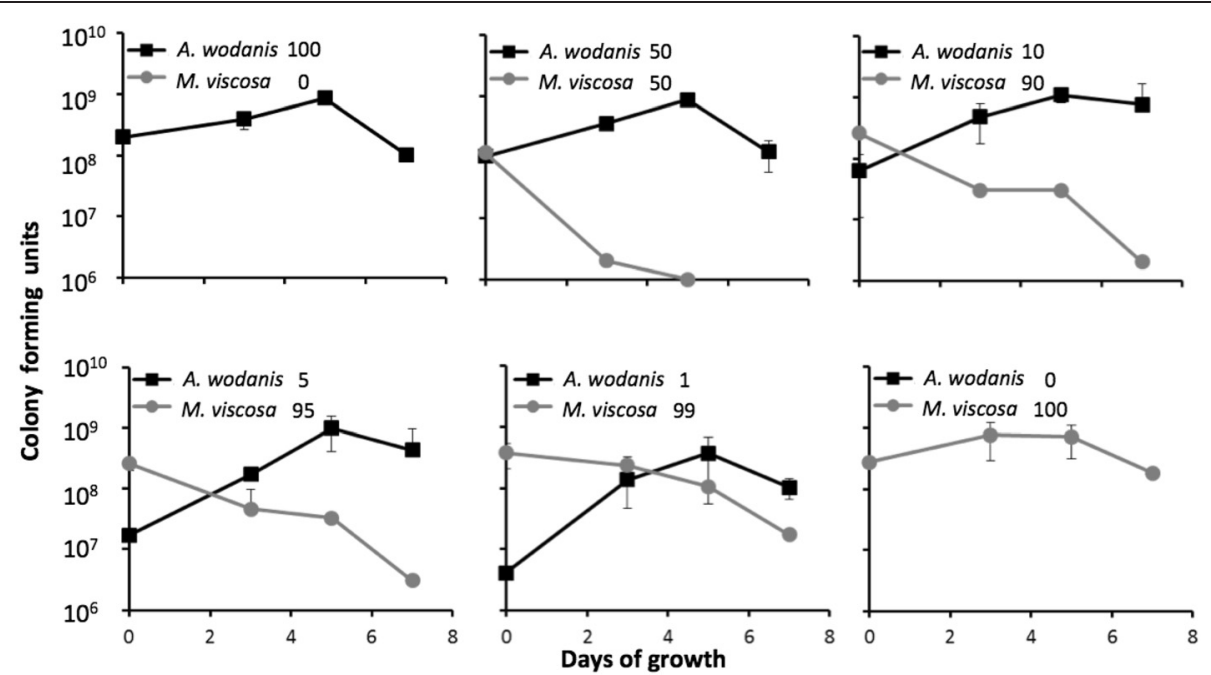

Fig. 2 Bacterial growth in mono- and co-culture on BA plates. The growth was measured by colony growth distribution from initial A. wodanis: M. viscosa culture mixtures with the following proportions; 100:0, 50:50, 10:90, 5:95, 1:99 and 0:100. The growth development within the colonies are estimates from two parallell experiments presented as the average \pm standard deviation of the colony forming units (cfu) determined. Samples were collected at initiation and after 3, 5 and 7 days

only managed to recover and isolate bacterial RNA from one biological replicate for each sample condition. The gene expression levels of both $M$. viscosa and A. wodanis are affected during co-infections relative to single infections, although the changes are most profound for $M$. viscosa. In co-infections versus single infections, $115 \mathrm{M}$. viscosa genes had two times lower expression value and p-value lower than 0.05 (Additional file 3a). A striking large number of these genes are involved in protein synthesis; 62 tRNAs, 11 rRNAs and three ribosomal proteins. The remaining genes encode various functions, some related to transcription, replication and stress adaptation; two DNA binding proteins, one sigma-54 modulation protein, one cell division protein, a universal stress protein, and a phage-shock protein. In total there were 49 genes with increased expression in co- versus single infections. Most of these genes have unknown functions, including the genes on plasmid pMVIS41 that seems to be up-regulated 2-4 fold when A. wodanis is present (Additional file $3 \mathrm{~b}$ ).

In co-infections, $87 \mathrm{~A}$. wodanis genes had two times lower expression ( $\mathrm{p}$-value $<0.05)$ relative to single infections. Similar to M. viscosa, many of these genes have functions related to protein synthesis and stress adaptation (Additional file 3c). In addition, 18 genes are probably exported or are membrane proteins. At the same time, $103 \mathrm{~A}$. wodanis genes were two times up-regulated in co-infections versus single infections. Over half of these genes (56 genes) are located on the four plasmids, most with unknown functions. Among up-regulated chromosomal genes there are several with functions related to transcription, including the RNA polymerase sigma 70 factor (Additional file 3d).
Species-specific genes-nutritional advantage

All enzymes and the metabolic pathways in both genomes were assessed using Priam. The two complete sets of enzymes were compared and mapped to KEGG using KEGG Mapper. In total $M$. viscosa and $A$. wodanis shared 873 enzymes, A. wodanis had 119 unique enzymes not shared with $M$. viscosa, while $M$. viscosa had 214 unique enzymes.

M. viscosa carry a potential siderophore biosynthesis operon of four genes (MVIS_0633-0636), including a gene with $75 \%$ similarity to Pseudomonas aeruginosa $p v d A$. This gene encodes L-ornithine N5-oxygenase, a key enzyme in the biosynthesis of the siderophore pyoverdin [17]. Siderophore biosynthesis, secretion and uptake in $A$. wodanis could be encoded by a locus (AWOD_I_1553-1563) that is highly similar and synthenic (Additional file 4) to the vibrioferrin locus of $V$. parahaemolyticus [18]. In addition A. wodanis carry a second siderophore uptake system (AWOD_II_0927-0923). The genes are over $90 \%$ similar at amino acids level to ferric anguibactin/vulnibactin uptake systems of several Moritella species, including M. marina [19].

\section{Inhibitorial factors}

The largest $A$. wodanis plasmid pAWOD920 carry six genes that could be involved in the production and secretion of bacteriocin (Fig. 3). The amino acid sequence of the putative bacteriocin (AWOD_p920_63) shows no similarity to any proteins in GenBank, but has the conserved GGmotif (Fig. 3) necessary for secretion [14]. The two subsequent genes AWOD_p920_61 and AWOD_p920_62 encode bacteriocin secretion proteins possible homologous 


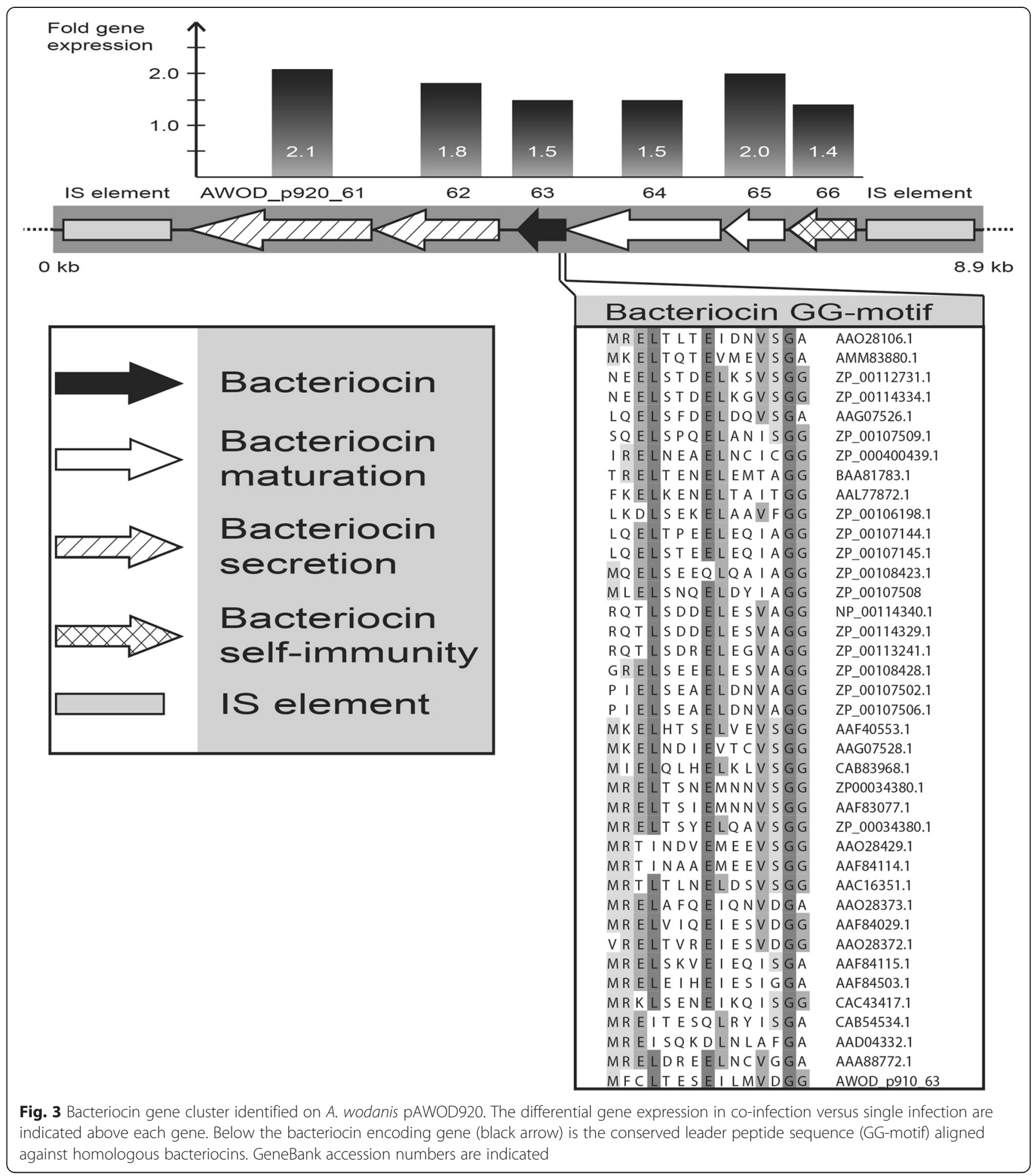

to Klebsiella pneumoniae RYC492 mceG and mceH. Their translated products are $62 \%$ and $46 \%$ similar, respectively. AWOD_p920_61 encodes a transport peptidase with the conserved $\mathrm{C} / \mathrm{H}$-motifs [(QX4[D/E]C[G/A]XAXLX2[I/ $\mathrm{V}] \mathrm{X} 4 \mathrm{GX} 4[\mathrm{I} / \mathrm{L}] \mathrm{X} 2 \mathrm{LR})$ and $(\mathrm{H}[\mathrm{Y} / \mathrm{F}][\mathrm{Y} / \mathrm{V}] \mathrm{V}[\mathrm{L} / \mathrm{V}] \mathrm{X} 9[\mathrm{I} / \mathrm{L} /$ $\mathrm{V}] \mathrm{XDP})$ ] and is probably responsible for cleaving off the bacteriocin leader sequence [20]. AWOD_p920_62 encode a HlyD family membrane fusion protein, which is thought to play a role in transport across the outer membrane. AWOD_p920_64 and AWOD_p920_65 may be homologs of $K$. pneumoniae RYC492 mceI and $m c e J$, both with similarities of $46 \%$ at the amino acid level, mceI and mceJ encode proteins involved in bacteriocin maturation [21]. AWOD_p920_66 encodes a 
CAAX protease belonging to the Abi family (PF02517) and may be related to self-immunity.

Prevalence of four bacteriocin-locus genes were assessed by PCR on a collection of $A$. wodanis isolates, which allowed the discrimination between bacteriocin-locus positive and negative isolates (Additional file 5). However, the three isolates that were found negative for the bacteriocin-locus produced PCR products from primers targeting bacteriocin secretion genes, although the product from one of the two genes were $\sim 300 \mathrm{bp}$ larger than expected (Additional file 5). Four bacteriocin carrying isolates, including $A$. wodanis $06 / 09 / 139$, were screened for bacteriocin production using four $M$. viscosa isolates as indicator organisms. In parallel, the same screening was performed on $A$. wodanis that did and that did not carry the genotypes (Additional file 5). Both on the blood agar (BA) streak and in the soft agar overlay assay, inhibitorial activity against several $M$. viscosa isolates were observed (Fig. 4 and Additional file 6). $M$. viscosa isolates also appear to be differently affected by A. wodanis, which is illustrated in Fig. 4a where M. viscosa isolates responds differently in growth to one $A$. wodanis strain. Growth inhibition was reproduced in the overlay assay (Fig. 4c) where the effect was more profound. The inhibitorial activity was also tested against $A$. wodanis isolates, and cross streaking revealed that the antimicrobial activity was strongest between $A$. wodanis isolates compared to the more distantly related $M$. viscosa isolates (Fig. 4ab, Additional file 6). A. wodanis isolates with the bacteriocin operon did not exert any strong inhibitorial effect on itself compared to $A$. wodanis without the bacteriocin operon when cross streaked simultaneously (Fig. 4b). However, a colony inhibited growth of the same strain when it was plated on the overlay agar (Fig. 4d) indicating that the older colony could inhibit growth on non-adapted cells of the same strain.

\section{Discussion}

When two different bacterial species are placed together in the same environment, there are at least three possible outcomes from this close encounter: The presence of a second bacterial species is not affecting the behaviour of the first; the two bacteria team up and aid each other in invading the nutrient source (for instance a host); or the two bacteria compete with each other for the same pool of resources.

The in vitro mono- and co-cultivation demonstrated that growth of the two bacteria is negatively affected by the presence of the other. Most profound was the impact $A$. wodanis had on M. viscosa growth. It is therefore likely that the relationship between the two species is of a competitive nature and that $A$. wodanis is better adapted to propagate when the two species occupies the same environment. This growth inhibition is not contact-dependent since the effect is similar when the two bacterial species are present in the same tubings as when they are in separate tubings.

Expression profiling of the transcriptome allows us to study the dynamic picture of regulatory and functional processes during adaptation to environmental changes. Gene expression profiles were measured when the bacteria were implanted together in the same fish and when they were implanted separately in different fishes. The effects $M$. viscosa and $A$. wodanis have on altering the global expression pattern in the presence of each other are discussed together with growth data from the mono- and co-culture growth experiments within the following sections. However, in the absence of biological replicates only careful interpretation of the general expression profiles are made.

\section{Growth and co-cultivation}

Multiple copies of ribosomal RNAs (rRNAs) are assumed to reflect the ability for bacteria to grow fast. Moreover, there is a correlation between the copy number of rRNA genes and the rate at which bacteria respond to resource availability [22]. Short replication time is an evolutionary adaptation advantageous in a competitive environment with limited access to nutrients. Although the M. viscosa genome is only $9 \%$ larger than A. wodanis, it contains almost twice the number of ribosomal RNA operons (11 versus 6 copies). From this one could assume that $M$. viscosa would grow faster than A. wodanis, however, under the growth conditions used in this study the opposite is the case (Fig. 1, Additional file 2).

In co-culture on blood agar, $A$. wodanis quickly becomes the dominant bacteria and outcompete $M$. viscosa, even when the initial ratio was 1:99 (Fig. 2). A similar impediment of $M$. viscosa growth is observed when they are co-cultured in LB medium (Fig. 1). In contrast, $A$. wodanis appear to be less affected when in co-culture (Figs. 1 and 2). The transcriptomic effect is strongest when the two bacteria are together in the same tubings, but the inhibition does not seem to be contactdependent as M. viscosa growth also is impeded when co-cultivated in separate tubings.

The general trends from the in vivo gene expression profiling of the transcriptomes was that genes with similar functions are down-regulated in both bacteria during co-infection relative to when they were implanted alone in the fish. We were not able to follow the growth rate of the bacteria in the implanted tubings and thus establish if any reduction in growth or if difference in gene expression is a result of nutritional deprivation of the environment. However, culture densities from growth in tubings (Fig. 1) and estimated cfu's on agar plates (Fig. 2) are still increasing for both $A$. wodanis and M. viscosa after four days of cultivation, which is the time point of when the implanted tubings were extracted from the 


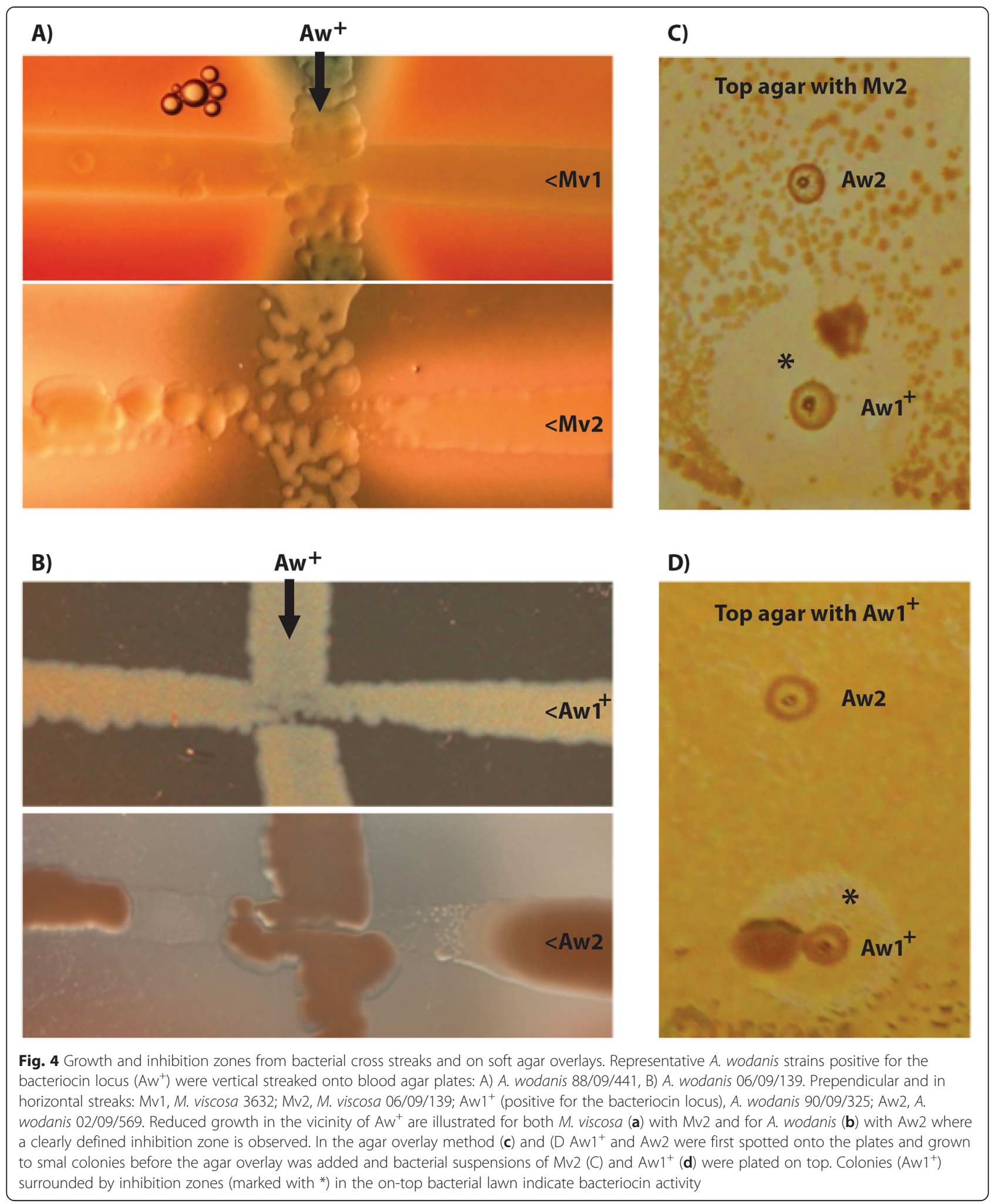

fish. The semipermeable membrane is also likely to diffuse factors (nutritional, immune components, etc.) that could influence growth within the abdominal cavity of Atlantic salmon. However, to determine effects of such interactions with the host is not within the scope of this study. In the presence of a putative competitive bacterial species, the RNA expression profiles suggest a negative gene regulation effect, particularly on genes involved in 
protein synthesis. Additionally, the expression of several genes related to stress adaptation, replication and transcription are also repressed in co-infections. The negative regulation of the protein synthesis is most profound for $M$. viscosa, where all 11 ribosomal RNAs and 106 out of the total 133 tRNAs (albeit only 53 genes with p-value $<0.05)$ are more than two fold down-regulated in the presence of $A$. wodanis. In addition, the expression of three ribosomal proteins is two fold lower in co-infections. The abundance of RNA polymerases and ribosomes are important growth rate-dependent parameters, and the rate-limiting step in ribosome synthesis is the synthesis of rRNA. When the nutritional availability is low, the synthesis of ribosomes decreases, which in turn slows protein production and lowers the growth rate in order to conserve the cell's energy (reviewed in [23]). It remains to be elucidated, but the reduced expression of $M$. viscosa genes involved in protein synthesis could be a result of $A$. wodanis being a more efficient in utilizing the nutrition available within the host environment, thereby preventing $M$. viscosa to meet its nutritional needs.

\section{Species-specific genes-nutritional advantage}

The abundance of iron in the marine environment is scarce. Also for pathogenic marine bacteria there is a constant battle for iron between the invading bacteria and the host. The uptake of iron is efficiently mediated through siderophores produced by the bacteria that scavenge extracellular iron [24]. The siderophore-iron complex is very beneficial when it is transported back into the cell, but it also comes at a cost; siderophore biosynthesis requires energy and the proper assembly of the components that constitutes the functional groups of the molecule.

Björnsdottir and colleagues showed that $M$. viscosa produces siderophores, but the method they used is universal and do not discriminate between various types of siderophores [25]. From the genome sequence alone we were not able to identify the genes responsible for the biosynthesis of these siderophores. However, the chromosome carries a four-gene operon similar to the siderophore pyoverdin biosynthesis genes, which is linked to biofilm formation in Pseudomonas aeruginosa [17]. The largest $A$. wodanis chromosome carries genes homologous to those responsible for vibrioferrin biosynthesis and uptake in $V$. parahaemolyticus [18]. A. wodanis also encodes a second siderophore uptake system, with similarities to anguibactin/vulnibactin acquisition systems found in other Moritella species [19], but lack the corresponding siderophore biosynthesis genes. Unlike the majority of marine siderophores, neither vibrioferrin nor anguibactin/ vulnibactin are amphiphilic siderophores [10], and are secreted from the cell to acquire iron. During an infection,
A. wodanis and M. viscosa have to compete against the host and each other to sequester iron. Siderophoremediated interspecies competition has been shown for other bacteria (reviewed in [9]). Since the two bacterial species seem to exhibit different types of siderophores, it is possible that $A$. wodanis produce siderophores with higher affinity for iron than $M$. viscosa. It is also possible that $A$. wodanis have retained the second siderophore uptake system as an agent to "steal" exogenous siderophores, hence putting the burden of producing siderophore on other species like $M$. viscosa. A similar strategy has been demonstrated with the ferric aerobactin receptor (IutA) mediated uptake of aerobactin by $V$. parahaemolyticus [26].

\section{Inhibitors}

Bacteria are able to outcompete other bacteria by secreting effector molecules. The impediment of $M$. viscosa growth in the presence of $A$. wodanis could be a result of inhibiting bacteriocin activity mediated by $A$. wodanis. Bacteriocin production has been reported for many members of the Vibrionaceae family ([20], [27], [28] and [29]), but the genotypes have to our knowledge not been determined. The A. wodanis plasmid pAWOD920 carries a locus of six genes (AWOD_p920_0066-0061) with all the functions necessary for the biosynthesis and export of bacteriocin (Fig. 3).

The majority of the genes located on A. wodanis plasmids have increased expression in co-infection relative to single infection. All the six genes in the bacteriocin locus are up-regulated 1.4-2.1 fold when $A$. wodanis was implanted together with $M$. viscosa in the same fish relative to when implanted separately in different fishes (Fig. 3). Whether this is a direct effect of gene regulation or the increase in plasmid copy numbers is not clear, but the translational outcome would be similar, and could give $A$. wodanis a competitive advantage over $M$. viscosa.

A. wodanis isolates had a strong inhibitorial effect on each other. From the cross streaking assay $A$. wodanis isolates carrying the bacteriocin-locus inhibited growth more strongly in $A$. wodanis isolates not predicted to carry this locus (Additional file 5). However, A. wodanis without this locus also inhibited growth on bacteriocinlocus carrying $A$. wodanis isolates. The PCR screen predicting the presence of the bacteriocin locus in $A$. wodanis isolates produced amplicons for genes predicted to secrete the bacteriocin precursor in all $A$. wodanis isolates tested (Additional file 5). A second bacteriocin system could be present in these isolates. The overlay assay displays the antimicrobial potential between both $A$. wodanis types including self-inhibition. This observation is presumably a consequence of that the older colony underneath the overlay plated bacteria is already producing the bacteriocin and the immunity protein, which confer resistance. The overlay-plated bacteria are not bacteriocin producing cells 
and have no immunity to the underlying bacteriocin producing cells. The inhibitorial effect is likely mediated through a diffusible agent. Both the growth inhibition zones around the colonies on the soft agar overlay and the reduced growth in the vicinity prior to the intersection point where the bacteria make physical contact on the BA plates (Fig. 4 and Additional file 6), demonstrates that the growth inhibition is not contact dependent.

Bacteriocin normally inhibits closely related bacterial species, although bacteriocins produced by other Vibrionaceaes have toxic effects on distantly related bacteria [12]. In accordance with this, the antimicrobial effect is stronger against other $A$. wodanis isolates than against $M$. viscosa (Fig. 4). Our results demonstrates that M. viscosa is susceptible to secreted agent(s) produced by $A$. wodanis, and that a likely candidate may be produced by the plasmid encoded bacteriocin locus.

\section{Quorum sensing}

When exploring the relationship between two bacteria, it is natural to consider the possibility that the interaction could be controlled through signal molecules of QS systems. QS-communication between different bacteria imply that the signal from one species must be detected and incorporated by the second species. Bacteria that have an incomplete QS circuit may retain the ability to detect and regulate gene transcription in response to AHL without having the ability to produce the molecule itself [30].

A. wodanis 06/09/139 is producing the AHL N-3hydroxy-decanoyl-L-homoserine lactone (3-OH-C10HSL) [31], while M. viscosa does not produce AHLs [32]. To our knowledge, production of AI-2 has not been assessed for these bacteria. In A. wodanis, the AinS/AinR (AWOD_I_1040/1039) system probably confers to the 3-OH-C10-HSL production. In addition, the genome also harbours a LuxS-LuxPQ (AWOD_I_0523AWOD_I_0676/0677) system. This system utilises AI-2 synthesised by LuxS. Both systems converge and mediate their response through a cascade of phosphorylation reactions of LuxU (AWOD_I_0921) and LuxO (AWOD_I_0920). This finally activates the master regulator LitR (AWOD_I_0419) which regulates the expression of a variety of genes including LuxR.

We were not able to identify any complete QS systems in M. viscosa, but genes homologous to litR (MVIS_3792) and luxR (MVIS_1035) were found. LuxR-family proteins respond to AHLs and can affect the expression of up to 625 target genes in Vibrio harveyi alone [33]. If any interspecies communication exists between these two species, the $M$. viscosa LuxR may act as a receptor for AHLs and possible AI-2 produced by $A$. wodanis. The effect of this potential eavesdropping is however not known.

\section{Winter-ulcer pathogenesis}

The winter-ulcer disease aetiology is complex, where "winter-ulcer" refers to infection with $M$. viscosa although additional species of bacteria such as $A$. wodanis and Tenacibaculum spp. are recurrently reported isolated. M. viscosa is highly virulent in Atlantic salmon [3] and secretes toxic compounds [25]. Skin has been suggested as the initial infection site as ulceration corresponds with direct skin surface colonization [34]. Also A. wodanis secretes toxins cytotoxic to fish cell lines, and is able to co-infect (with M. viscosa) Atlantic salmon [3]. Likewise is a Tenacibaculum sp. shown to produce skin ulcers in scarified areas during challenge and coinfect ulcers already induced by M. viscosa [35]. This indicates the presence of multimicrobial communities on fish skin or in ulcers, which are likely to interact with each other. Predisposing Atlantic salmon to A. wodanis before $M$. viscosa challenge reduce mortality compared to mono-infection with $M$. viscosa [3]. In this study, $A$. wodanis is also shown to affect $M$. viscosa growth. The encoding bacteriocin locus in $A$. wodanis could in part be the responsible factor that negatively impedes $M$. viscosa growth and thereby indirectly reduce $M$. viscosa virulence. Such a reduction in virulence could relate to the chronic development of the winter-ulcer disease observed in field outbreaks compared to the acute phase development in experimental settings where only $M$. viscosa is present. Overall this could imply that the microbial composition present on the surface of a fish is influencing the pathogenesis of skin-mucosal diseases such as winterulcer, and that modulating this layer is a potential mechanism to influence the pathogenesis of diseases.

\section{Conclusions}

The two bacteria $M$. viscosa $06 / 09 / 139$ and $A$. wodanis 06/09/139 were isolated from one Atlantic salmon with winter-ulcer disease and the genomes sequenced. Cocultivation studies showed that $A$. wodanis is impeding the growth of $M$. viscosa, and that the inhibitorial effect is not contact-dependent. The competition could either come from the fight over nutritional resources, or it could come from that $A$. wodanis is actively producing and secreting bacteriocin-like agents that impede growth across species including both $A$. wodanis and M. viscosa. Expression profiles of the transcriptomes supports that the presence of $A$. wodanis affect $M$. viscosa, possibly by altering the global gene expression of $M$. viscosa. Further studies are needed to understand the interplay between $M$. viscosa and $A$. wodanis in causing winter-ulcer disease in fish. The overall findings reported in this study can be useful for future development of alternative bioproducts that could potentially control the survival and health status of farmed fish. 


\section{Methods}

\section{Strains and growth conditions}

A. wodanis strain 06/09/139 and M. viscosa strain 06/09/ 139 , originally isolated from the head kidney of an Atlantic salmon diagnosed with winter-ulcer disease and previously confirmed pathogenic to Atlantic salmon [3] were streaked out onto blood agar (BA) plates (containing blood agar base no. 2, Oxoid CM271, 7 \% human whole blood and $2.5 \%(\mathrm{w} / \mathrm{v}) \mathrm{NaCl})$. Single colonies were selected and expanded for two days at $200 \mathrm{rpm}$ shaking in Luria-Bertani broth (LB) with $1.0 \% \mathrm{NaCl}$ (LB1) at $7{ }^{\circ} \mathrm{C}$. In an attempt to mimic their co-existence and growth as observed from field outbreaks, bacteria were grown in tubings in vitro, either in mono-culture or in co-culture. Bacteria cultures were diluted to $\mathrm{A}_{600} \sim 0.015$ in LB1 and cultivated in sterile semi-permeable $25 \mathrm{~mm}$ diameter regenerated cellulose dialysis tubings with MW cut-off 12-14 000 Da (Sprectra/Por, Los Angeles, CA), immersed in an equal volume of $200 \mathrm{ml} \mathrm{LB1}$. Co-culture growth was also assessed by colony growth distribution. M. viscosa and $A$. wodanis grown in LB1 at $7{ }^{\circ} \mathrm{C}$ to approximately $\mathrm{A}_{600} \sim 1.0$ were diluted to $10^{-1}$ suspensions before assorted co-culture mixtures with increasing $M$. viscosa and decreasing A. wodanis ratios were made. The co-cultures were spotted in $2 \mu \mathrm{l}$ aliquots onto BA plates containing $1 \% \mathrm{NaCl}$ and incubated at $7{ }^{\circ} \mathrm{C}$. Growth rates in spotted co-culture colonies were examined at initiation and at day 3,5 and 7 by resuspending entire colonies in LB1 and plating ten-fold serial dilutions onto BA plates.

\section{Inoculation of dialysis tubings and in vivo implantation}

Preparation of dialysis tubings was performed after the method of Colquhoun et al. (1998) [36]. Briefly, $20 \mathrm{~cm}$ of dialysis tubings (described above) were autoclaved in PBS and added $1 \mathrm{ml}$ (OD 0.6) bacterial suspension cultured in LB1. The model utilized three sets of groups; mono-cultures of either M. viscosa or A. wodanis in one tube was implanted, the second set where $M$. viscosa and $A$. wodanis were implanted together in separate tubes, and the third set where $M$. viscosa and $A$. wodanis were implanted together in the same tube. Three consecutive knots sealed with silicon followed by ethanol desinfection prevented leakage. The implants were stored in sterile LB1 until implantation (within two hours). Unvaccinated Atlantic salmon obtained from Akvaforsk AS (Sunndalsøra, Sjølseng 12917, Norway) were maintained at the Norwegian Institute for Water Research's aquaculture station (Solbergstrand, Norway). Fish $(\sim 1 \mathrm{~kg})$ were anesthetised $(0.0025 \%$ benzocaine solution, Sigma, St. Louis, MO), before executing a small incision (appr. $2 \mathrm{~cm}$ long) medially and anteriorly to the pelvic fins. Dialysis tubings were fed into the peritoneal cavity followed by closure of the incision with three sutures (Ethicon, Suturamid 4-0), each passing through all layers of tissue.
A solution of benzocaine and fresh seawater was pipetted over the gills periodically during the surgery. All fish survived the surgery and implanted fish were marked by fin clipping. The fish were kept in a $1400 \mathrm{~L}$ tank of seawater (salinity $3.1-3.5 \%$ ) at $9{ }^{\circ} \mathrm{C}$ without feeding. The water quality was monitored throughout the experiment and the oxygen content was maintained stable at $8.2 \mathrm{mg} \mathrm{l-1}$. Fish were euthanized on day 4 with an overdose of benzocaine and a blow to the head. Bacterial cultures from the recovered implants were briefly centrifuged (14 $000 \times \mathrm{g}, 2 \mathrm{~min}$, $4{ }^{\circ} \mathrm{C}$ ) before snap frozen in liquid nitrogen (within $3 \mathrm{~min}$ after removal from the fish).

\section{Whole genome sequencing}

The two M. viscosa 06/09/139 and A. wodanis 06/09/139 genomes have been completely sequenced using a combination of 454, Illumina and Pacific Bioscience sequencing technology. Total bacterial DNA was isolated using proteinase $\mathrm{K}$ treatment followed by chloroform:phenol extraction. Initially, the 454 data was de novo assembled with Newbler [37], and further used as a scaffold for the assembly of the Illumina data using IMAGE [38]. The scaffolds were ordered with regards to the complete genomes of closely related species using ABACAS [39], and remaining gaps closed by sequencing positive PCR products bridging contigs in the assembly.

\section{Functional annotation and pathway analysis}

Both genomes have been functionally annotated as described previously [40] using Artemis software [41], and are available from EMBL/GenBank/DDBJ with the accession numbers PRJEB6964 and PRJEB6963. Additionally, Priam [42] and KEGG Mapper [43] was used to predict enzymes and visualize metabolic pathways, respectively.

\section{RNA preparation and CDNA synthesis}

RNA was isolated using the MasterPure ${ }^{\mathrm{Tm}}$ Complete DNA and RNA Purification Kit (EPICENTER, Cat. Nos. MC85200 and MC89010) according to manufacturer's instructions. RNA samples were quantified by ND-1000 Spectrophotometer (NanoDrop ${ }^{\oplus}$ Technology, DE, USA) and its integrity controlled by agarose gel electrophoresis in all successive steps. rDNase I included in the DNA-Free ${ }^{\mathrm{Tm}}$ Kit (Ambion) was used to remove excess genomic DNA according to manufacturer's instructions. Samples were confirmed DNA-free with a $16 \mathrm{~S}$ rDNA primer set to a level below PCR detection and inactive for DNase by digestion analysis. Twenty five $\mu \mathrm{g}$ of each total RNA extract was reversely transcribed using random hexamers, 40U RNaseOUT ${ }^{\mathrm{TM}}$ and $400 \mathrm{U}$ Superscript III RT (Invitrogen) at $42{ }^{\circ} \mathrm{C}$ for two hours. Denaturation occurred at $70{ }^{\circ} \mathrm{C}$ for $20 \mathrm{~min}$. Transcription products were purified using Illustra AutoSeq G-50 
columns (GE Healthcare) and by Trizol (GibcoBRL) extraction. cDNA synthesis was confirmed by PCR.

\section{Library construction and sequencing}

The single-stranded cDNA libraries were constructed as described in [44]. Gel electrophoresis was used to select for DNA constructs approximately $200 \mathrm{bp}$ in size, and amplified by PCR. The libraries were loaded onto two separate lanes of an Illumina GA flow cell and sequencing reactions performed according to the manufacturer's recommended protocol.

\section{Read mapping and visualisation}

The cDNA sequence reads were mapped uniquely to the genomes of $M$. viscosa 06/09/139 and A. wodanis 06/09/139, and quantification of transcript abundance and calculation of differential gene expression were performed using Rockhopper [45]. Gene expression levels were quantified as the number of sequence reads mapping to the sequence of the gene divided by the length of the gene and the upper quartile of gene expression. Rockhopper use the Negative Binomial distribution as a model to compute $p$-values, and we regarded $p>0.05$ as the probability of observing a transcript's expression levels in different conditions by chance.

\section{PCR screening and bacteriocin assay}

Total DNA preparation from all bacterial isolates, PCR amplification and amplicon visualization (Additional file 5) of the putative plasmid bacteriocin operon was performed identical to [3].

A. wodanis isolates, listed in Additional file 5, were screened for production of bacteriocin. Isolates were grown in $3 \mathrm{ml} \mathrm{LB} 3.5 \% \mathrm{NaCl}$ at $10{ }^{\circ} \mathrm{C}$ with shaking. Cultures $(100 \mu \mathrm{l})$ were diluted and further expanded for two days in $3 \mathrm{ml} \mathrm{LB} 1$ at $10{ }^{\circ} \mathrm{C}$ with shaking. Cultures were adjusted to $\mathrm{OD}_{600} \sim 0.4$ and $100 \mu \mathrm{l}$ were inoculated in $3 \mathrm{ml}$ fresh LB1 at $10{ }^{\circ} \mathrm{C}$ and grown to approximately $\mathrm{A}_{600} \sim 0.25$ to 0.35 . A culture loop $(1 \mu \mathrm{l})$ of one of the bacterial suspensions was then streaked in a single straight line in the centre of BA plates (containing a total of $1.0 \%$ $\mathrm{NaCl}$ and supplemented with $5 \%$ bovine blood). A loopfull from each culture was streaked perpendicular to this traversing the original streaked suspension. This method was followed for all isolates. Plates were incubated at $8{ }^{\circ} \mathrm{C}$ for 5 days before being examined for growth inhibition patterns. To confirm antimicrobial activity production $2 \mu \mathrm{l}$ of the bacterial suspensions were spotted onto Luria agar containing $1.0 \% \mathrm{NaCl}$ (LA1) incubated at $8{ }^{\circ} \mathrm{C}$ for 3 days. Plates and colonies were then overlaid with a new layer of LA1 and $10^{3}$ serial diluted bacterial suspensions $\left(\mathrm{A}_{600} \sim 0.4\right)$ were plated on top. Plates were incubated 5 days at $8{ }^{\circ} \mathrm{C}$. Colonies surrounded by clear zones in the on-top bacterial lawn indicated bacteriocin activity.

\section{Ethical approval}

The study "In vivo communication between winter ulcer bacteria Moritella viscosa and Vibrio wodanis", Id. Nr 2041 was approved by the Norwegian National Animal Research Authority in August 26. 2009.

\section{Availability of supporting data}

The annotated genomes have been deposited to the European Nucleotide Archive ENA (http://www.ebi. ac.uk/ena/) with the accession numbers PRJEB6964 and PRJEB6963.

The Illumina reads from the RNA sequencing have been deposited to the Sequence Read Archive repository at ENA and they are available under study accession number ERP006698, and the meta data to ArrayExpress under accession number E-MTAB-2851.

\section{Additional files}

\begin{abstract}
Additional file 1: Schematic circular diagrams of the replicons of $A$ ) M. viscosa 06/09/139 and B) A. wodanis 06/09/139. Appropriate

categories are shown as pairs of concentric circles representing both coding strands. Key to the chromosomal circular diagrams (outside to inside): scale (in Mb), annotated CDS, pseudogenes (brown), non-coding and structural RNA; rRNA (blue), tRNA (green) and sRNA (pink), \% G + C content, $\mathrm{G}+\mathrm{C}$ deviation ( $>0 \%$ olive, $<0 \%$ purple). Colour coding for CDSs (according to predicted function): dark blue, pathogenicity/adaptation; black, energy metabolism; red, information transfer; dark green, surface associated; cyan, degradation of large molecules; magenta, degradation of small molecules; yellow, central/intermediary metabolism; pale green, unknown; pale blue, regulators; orange, conserved hypothetical; brown, pseudogenes; pink, phage + IS elements; grey, miscellaneous.
\end{abstract}

Additional file 2: Growth curves of $A$. wodanis 06/09/139 and $M$. viscosa $06 / 09 / 139$ at $7{ }^{\circ} \mathrm{C}$. Colonies from blood agar plates were expanded over-night in $\mathrm{LB}$ with $3.5 \% \mathrm{NaCl}$ at $7{ }^{\circ} \mathrm{C}$. The cultures were used to inoculate fresh LB with either $1.0 \%$ or $3.5 \% \mathrm{NaCl}$ to a concentration of $A_{600} \sim 0.02$. Cultures were expanded to exponential phase ( $A$. wodanis $A_{600} \sim 0.8, M$. viscosa $\left.A_{600} \sim 0.4\right)$ and further used to inoculate fresh LB with either $1.0 \%$ or $3.5 \% \mathrm{NaCl}$ to a concentration of $\mathrm{A}_{600} \sim 0.02$. Density measurements were obtained regularly for triplicates of each condition and is presented as $A_{600}$ average \pm standard deviation.

Additional file 3: $M$. viscosa and $A$. wodanis genes that are more than 2 -fold up- or down regulated ( $p$-value $<0.05$ ) in co-infection versus single infection.

Additional file 4: Comprison of the siderophore biosynthesis, secretion and uptake locus in $A$. wodanis 06/09/139 and the vibrioferrin locus of $V$. parahaemolyticus WP1. Similarities of the translated products are shown in grey.

Additional file 5: $A$. wodanis isolates screened for the presence of bacteriocin locus genes.

Additional file 6: Antimicrobial activity assays by cross streaking isolates on blood agar plates and by observing inhibition zones on the soft agar overlay.

\section{Abbreviations}

QS: Quorum sensing; AHL: N-acyl homoserine lactone; BA: Blood agar; cfu: colony forming unit.

\section{Competing interests}

The authors declare that they have no competing interests. 


\section{Authors' contributions}

EH, CK, HS, NRT, and NPW conceived and designed the experiments. HS provided the bacterial strains used in this study, made the in vivo implantation, and together with NPW supervised the project, contributing ideas and experimental design. CK made the bacterial impants (tubings) and performed all DNA and RNA extrations both from the in vivo and in vitro part of the study. Further, CK carried out all growth experiments and the bacteriocin screening. With aid from NRT, EH analyzed the genomic DNA and the RNA sequencing data. EH and CK drafted the manuscript, EH, CK, HS, NRT, and NPW edited the manuscript. All authors read and approved the manuscript.

\section{Acknowledgement}

We greatly appreciate the contribution of Nick Chroucher, who aided the initial analysis of the RNA sequence data. This work was supported by MABIT (Marine Biotechnology in Tromsø).

\section{Author details}

${ }^{1}$ Department of Chemistry, Faculty of Science and Technology, University of Tromsø, N-9037 Tromsø, Norway. ${ }^{2}$ Section of Microbiology, Immunology and Parasitology, Department of Food Safety and Infection Biology, Faculty of Veterinary Medicine and Biosciences, Norwegian University of Life Sciences, Ullevålsveien 72, Oslo, Norway. ${ }^{3}$ The Pathogen Sequencing Unit, The Wellcome Trust Sanger Institute, Wellcome Trust Genome Campus, Hinxton, Cambridge CB10 1SA, UK. ${ }^{4}$ The Norwegian Structural Biology Centre, University of Tromsø, N-9037, Tromsø, Norway.

\section{Received: 16 September 2014 Accepted: 29 May 2015}

\section{Published online: 10 June 2015}

\section{References}

1. Lunder T, Evensen $\varnothing$, Holstad G, Håstein T. Winter ulcer in the Atlantic salmon Salmo salar. Pathological and bacteriological investigations and transmission experiments. Dis Aquat Organ. 1995;23:39-49.

2. Lunder T, Sorum H, Holstad G, Steigerwalt AG, Mowinckel P, Brenner DJ. Phenotypic and genotypic characterization of Vibrio viscosus sp. nov. and Vibrio wodanis sp. nov. isolated from Atlantic salmon (Salmo salar) with 'winter ulcer'. Int J Syst Evol Microbiol. 2000;2:427-50.

3. Karlsen C, Vanberg C, Mikkelsen H, Sorum H. Co-infection of Atlantic salmon (Salmo salar), by Moritella viscosa and Alivibrio wodanis, development of disease and host colonization. Vet Microbiol. 2014;171(1-2):112-21.

4. Kuramitsu HK, He X, Lux R, Anderson MH, Shi W. Interspecies interactions within oral microbial communities. Microbiol Mol Biol Rev. 2007;71(4):653-70.

5. Kolenbrander PE. Multispecies communities: interspecies interactions influence growth on saliva as sole nutritional source. Int J Oral Sci. 2011;3(2):49-54

6. Camilli A, Bassler BL. Bacterial small-molecule signaling pathways. Science. 2006;311(5764):1113-6.

7. Dyszel JL, Soares JA, Swearingen MC, Lindsay A, Smith JN, Ahmer BM. E. coli K-12 and EHEC genes regulated by SdiA. PLoS One. 2010;5(1):e8946.

8. Federle MJ. Autoinducer-2-based chemical communication in bacteria: complexities of interspecies signaling. Contrib Microbiol. 2009;16:18-32.

9. Hibbing ME, Fuqua C, Parsek MR, Peterson SB. Bacterial competition: surviving and thriving in the microbial jungle. Nat Rev Microbiol. 2010;8(1):15-25.

10. Sandy M, Butler A. Microbial iron acquisition: marine and terrestrial siderophores. Chem Rev. 2009;109(10):4580-95.

11. Joshi F, Archana G, Desai A. Siderophore cross-utilization amongst rhizospheric bacteria and the role of their differential affinities for Fe3+ on growth stimulation under iron-limited conditions. Curr Microbiol. 2006;53(2):141-7.

12. Cascales E, Buchanan SK, Duche D, Kleanthous C, Lloubes R, Postle K, et al. Colicin biology. Microbiol Mol Biol Rev. 2007;71(1):158-229.

13. Datta A, Prescott LM. Effect of vibriocins on members of the Enterobacteriaceae. J Bacteriol. 1969;98(2):849-50.

14. Michiels J, Dirix G, Vanderleyden J, Xi C. Processing and export of peptide pheromones and bacteriocins in Gram-negative bacteria. Trends Microbiol. 2001;9(4):164-8
15. Haft DH, Basu MK, Mitchell DA. Expansion of ribosomally produced natural products: a nitrile hydratase- and Nif11-related precursor family. BMC Biol. 2010;8:70.

16. Kjos M, Snipen L, Salehian Z, Nes IF, Diep DB. The abi proteins and their involvement in bacteriocin self-immunity. J Bacteriol. 2010;192(8):2068-76.

17. Visca P, Ciervo A, Orsi N. Cloning and nucleotide sequence of the pvdA gene encoding the pyoverdin biosynthetic enzyme L-ornithine N5-oxygenase in Pseudomonas aeruginosa. J Bacteriol. 1994;176(4):1128-40.

18. Tanabe T, Funahashi T, Nakao H, Miyoshi S, Shinoda S, Yamamoto S. Identification and characterization of genes required for biosynthesis and transport of the siderophore vibrioferrin in Vibrio parahaemolyticus. J Bacteriol. 2003;185(23):6938-49.

19. Kautharapu KB, Jarboe LR. Genome sequence of the psychrophilic deep-sea bacterium Moritella marina MP-1 (ATCC 15381). J Bacteriol. 2012;194(22):6296-7.

20. Dirix G, Monsieurs P, Dombrecht B, Daniels R, Marchal K, Vanderleyden J, et al. Peptide signal molecules and bacteriocins in Gram-negative bacteria: a genome-wide in silico screening for peptides containing a double-glycine leader sequence and their cognate transporters. Peptides. 2004;25(9):1425-40.

21. Lagos R, Baeza M, Corsini G, Hetz C, Strahsburger E, Castillo JA, et al. Structure, organization and characterization of the gene cluster involved in the production of microcin E492, a channel-forming bacteriocin. Mol Microbiol. 2001;42(1):229-43.

22. Klappenbach JA, Dunbar JM, Schmidt TM. rRNA operon copy number reflects ecological strategies of bacteria. Appl Environ Microbiol. 2000;66(4):1328-33.

23. Gourse RL, Gaal T, Bartlett MS, Appleman JA, Ross W. rRNA transcription and growth rate-dependent regulation of ribosome synthesis in Escherichia coli. Annu Rev Microbiol. 1996;50:645-77.

24. Griffiths GL, Sigel SP, Payne SM, Neilands JB. Vibriobactin, a siderophore from Vibrio cholerae. J Biol Chem. 1984;259(1):383-5.

25. Bjornsdottir B, Gudmundsdottir T, Gudmundsdottir BK. Virulence properties of Moritella viscosa extracellular products. J Fish Dis. 2011;34(5):333-43.

26. Funahashi T, Tanabe T, Aso H, Nakao H, Fujii Y, Okamoto K, et al. An iron-regulated gene required for utilization of aerobactin as an exogenous siderophore in Vibrio parahaemolyticus. Microbiology. 2003;149(Pt 5):1217-25.

27. Carraturo A, Raieta K, Ottaviani D, Russo GL. Inhibition of Vibrio parahaemolyticus by a bacteriocin-like inhibitory substance (BLIS) produced by Vibrio mediterranei 1. J Appl Microbiol. 2006;101(1):234-41.

28. Shehane SD, Sizemore RK. Isolation and preliminary characterization of bacteriocins produced by Vibrio vulnificus. J Appl Microbiol. 2002;92(2):322-8.

29. Balakrishnan B, Ranishree JK, Thadikamala S, Panchatcharam P. Purification, characterization and production optimization of a vibriocin produced by mangrove associated Vibrio parahaemolyticus. J Coastal Life Med. 2013;1(3):169-77.

30. Dyszel JL, Smith JN, Lucas DE, Soares JA, Swearingen MC, Vross MA, et al. Salmonella enterica serovar Typhimurium can detect acyl homoserine lactone production by Yersinia enterocolitica in mice. J Bacteriol. 2010;192(1):29-37.

31. Purohit AA, Johansen JA, Hansen H, Leiros HK, Kashulin A, Karlsen C, et al. Presence of acyl-homoserine lactones in 57 members of the Vibrionaceae family. J Appl Microbiol. 2013;115(3):835-47.

32. Bruhn JB, Dalsgaard I, Nielsen KF, Buchholtz C, Larsen JL, Gram L. Quorum sensing signal molecules (acylated homoserine lactones) in gram-negative fish pathogenic bacteria. Dis Aquat Organ. 2005;65(1):43-52.

33. van Kessel JC, Rutherford ST, Shao Y, Utria AF, Bassler BL. Individual and combined roles of the master regulators AphA and LuXR in control of the Vibrio harveyi quorum-sensing regulon. J Bacteriol. 2013;195(3):436-43.

34. Karlsen C, Sorum H, Willassen NP, Asbakk K. Moritella viscosa bypasses Atlantic salmon epidermal keratocyte clearing activity and might use skin surfaces as a port of infection. Vet Microbiol. 2012;154(3-4):353-62.

35. Olsen AB, Nilsen $H$, Sandlund N, Mikkelsen $H$, Sorum $H$, Colquhoun DJ. Tenacibaculum sp. associated with winter ulcers in sea-reared Atlantic salmon Salmo salar. Dis Aquat Organ. 2011;94(3):189-99.

36. CD J, Sorum H. Outer membrane protein expression during in vivo cultivation of Vibrio salmonicida. Fish Shellfish Immunol. 1998:8(5):367-77.

37. Margulies M, Egholm M, Altman WE, Attiya S, Bader JS, Bemben LA, et al. Genome sequencing in microfabricated high-density picolitre reactors. Nature. 2005;437(7057):376-80. 
38. Tsai IJ, Otto TD, Berriman M. Improving draft assemblies by iterative mapping and assembly of short reads to eliminate gaps. Genome Biol. 2010;11(4):R41.

39. Assefa S, Keane TM, Otto TD, Newbold C, Berriman M. ABACAS: algorithm-based automatic contiguation of assembled sequences. Bioinformatics. 2009;25(15):1968-9.

40. Hjerde E, Lorentzen MS, Holden MT, Seeger K, Paulsen S, Bason N, et al. The genome sequence of the fish pathogen Aliivibrio salmonicida strain LFI1238 shows extensive evidence of gene decay. BMC Genomics. 2008;9:616.

41. Carver T, Harris SR, Berriman M, Parkhill J, McQuillan JA. Artemis: an integrated platform for visualization and analysis of high-throughput sequence-based experimental data. Bioinformatics. 2012;28(4):464-9.

42. Claudel-Renard C, Chevalet C, Faraut T, Kahn D. Enzyme-specific profiles for genome annotation: PRIAM. Nucleic Acids Res. 2003;31(22):6633-9.

43. Kanehisa M, Goto S, Sato Y, Furumichi M, Tanabe M. KEGG for integration and interpretation of large-scale molecular data sets. Nucleic Acids Res. 2012:40(Database issue):D109-14.

44. Croucher NJ, Fookes MC, Perkins TT, Turner DJ, Marguerat SB, Keane T, et al. A simple method for directional transcriptome sequencing using Illumina technology. Nucleic Acids Res. 2009;37(22), e148.

45. McClure R, Balasubramanian D, Sun Y, Bobrovskyy M, Sumby P, Genco CA, et al. Computational analysis of bacterial RNA-Seq data. Nucleic Acids Res. 2013;41(14), e140.

\section{Submit your next manuscript to BioMed Central and take full advantage of:}

- Convenient online submission

- Thorough peer review

- No space constraints or color figure charges

- Immediate publication on acceptance

- Inclusion in PubMed, CAS, Scopus and Google Scholar

- Research which is freely available for redistribution 